\title{
Sarcomeric model of stretch-induced stress fiber reorganization
}

This article was published in the following Dove Press journal:

Cell Health and Cytoskeleton

29 December 2010

Number of times this article has been viewed

\author{
Roland Kaunas' \\ Hui-Ju Hsu' \\ Shinji Deguchi \\ 'Department of Biomedical \\ Engineering, Texas A\&M University, \\ College Station, TX, USA; \\ ${ }^{2}$ Department of Biomedical \\ Engineering, Tohoku University, \\ Sendai, Japan
}

\begin{abstract}
Actin stress fibers (SFs) are mechanosensitive structural elements that respond to applied stress and strain to regulate cell morphology, signal transduction, and cell function. Results from various studies indicate that SFs tend to maintain stress or strain at a constant level. We developed a simple quantitative sarcomeric model of SFs to predict the role of actomyosin crossbridge cycling in SF tension regulation and reorientation in response to cyclic stretching. Under static conditions, the steady-state levels of SF tension were determined by the fiber passive stiffness and the stall force of the constituent myosin II filaments. When subject to cyclic changes in length at low frequencies, SFs change their unloaded reference length levels through myosin sliding to maintain tension at the original level. At high stretch frequencies, myosin cannot respond quickly enough and the SF behaves elastically. Myosin sliding also contributes to SF turnover, resulting in SF reorientation away from the direction of stretching at high, but not low, stretch frequencies. Using model parameters extracted from the literature, our model describes the dependence of cyclic stretch-induced SF alignment on stretch frequency and pattern consistent with experimental findings. This analysis predicts that myosin II plays multiple roles in regulating the ability of SFs to adapt to a dynamic mechanical environment.
\end{abstract}

Keywords: mechanical stretch, cytoskeletal dynamics, myosin, mechanotransduction

\section{Introduction}

Actin stress fibers (SFs) are contractile bundles that form in response to myosindependent contractility and applied forces such as mechanical stretch. ${ }^{1}$ Intracellular tension and cyclic stretching have been found to regulate the differentiation of somatic and stem cells. ${ }^{2-4}$ Further, increases in cell tension can enhance cell proliferation, ${ }^{5}$ which has important implications in normal tissue maintenance ${ }^{6}$ and in disease states such as cancer. ${ }^{7}$ Therefore, it is important to better understand how cells regulate tension.

SFs in nonmuscle cells consist of bundles of actin filaments cross-linked mainly by $\alpha$-actinin and myosin II. ${ }^{8,9}$ Myosin II and $\alpha$-actinin appear alternately along SFs, accompanied by periodic alterations in actin polarity. ${ }^{8}$ These findings led to early models of SF structure and contraction very similar to the organization of muscle sarcomeres. ${ }^{9}$ In electron micrographs, bands of $\alpha$-actinin in SFs are observed as electron-dense striations, with myosin-rich regions appearing as interleaving electron light regions. ${ }^{10}$ In a sarcomeric model of SF contraction, intercalating arrays of myosin slide between adjacent actin filaments to result in overall shortening or lengthening of the SF during concentric and eccentric contractions, respectively. Large ventral SFs are anchored to the substrate at each end via focal adhesions. ${ }^{11,12}$ Consequently, myosin-generated contraction leads to the development of isometric tension or 
prestress. ${ }^{13}$ This prestress extends SFs beyond their unloaded lengths. ${ }^{14,15}$ The level of SF pre-extension is maintained at a set point value with measured values ranging from 1.10 to 1.35. ${ }^{15}$ In vivo, cells are subject to deformations that perturb the cellular level of stress and strain. In collagen matrices, resident cells react to changes in tension in a direction toward re-establishment of a set point value in a process that Brown et al termed "tensional homeostasis". ${ }^{16}$

Despite the similarity in sarcomeric structure, sarcomeres in SFs and myofibrils differ in many respects. Nonmuscle myosin II is important for SF disassembly in cell processes requiring actin cytoskeletal turnover, such as cell crawling. ${ }^{17}$ In contrast, sarcomeres in myofibrils are very stable structures, which can be attributed to additional supporting proteins as well as the large number of myosin heads available for binding with actin filaments. Myosin filaments in SFs consist of 8-10 myosin proteins at each end of a filament, whereas those in myofibrils contain $\sim 150$ per half a myosin filament. ${ }^{18-20}$ The fraction of myosin heads bound to actin in SFs, ie, the duty ratio, is reported to be only $\sim 0.05 .{ }^{19,21}$ Any further decrease in the duty ratio, such as through decreased myosin ATPase activity, ${ }^{19}$ could therefore lead to the complete detachment of a myosin filament from adjacent actin filaments. Indeed, we demonstrated that raising the concentrations of MgATP from $<2$ to $4 \mathrm{mM}$ causes transition of SFs from concentric shortening to rapid SF disassembly in cytosol-free semi-intact cells. ${ }^{22}$

Rapid crossbridge cycling, such as occurs during SF contraction, also lowers the duty ratio. ${ }^{19}$ In the case of eccentric contraction, studies using myosin II-coated beads indicate that forces greater than the stall force for myosin cause backward slipping through transient detachment of actomyosin crossbridges, with more detachments leading to faster slipping. ${ }^{23}$ Thus, myosin sliding caused by either concentric or eccentric contractions are expected to reduce the duty ratio and hence decrease the stability of an SF due to fewer actomyosin cross-links. Consistent with this hypothesis, cyclic stretching of nonmuscle cells induces the gradual alignment of SFs in directions that minimize cyclic changes in SF length. ${ }^{24,25}$ The extent of SF alignment depends on the magnitude ${ }^{1}$ and frequency ${ }^{26}$ of stretching. We demonstrated that the dynamic reorganization of SFs could be described with a phenomenological model of SF relaxation and turnover. ${ }^{26}$ We now analyze SF behavior using a sarcomeric model to demonstrate that the force-dependent rate of myosin movement along actin filaments provides a molecular mechanism for the relaxation of perturbations in SF tension in response to changes in SF length. Further, we predict that stretch-induced decreases in SF stability due to myosin movement can describe the time-dependent alignment of SF in response to different frequencies and patterns of stretch.

\section{Model description and results Model of a stress fiber}

The present mathematical model of an SF in nonmotile cells is based on a sarcomeric description of SFs. Figure $1 \mathrm{~A}$ is a schematic representation of an individual SF that is firmly adhered to a surface at each end through transmembrane focal adhesion protein complexes in a nonmotile cell. The SF consists of a series of identical sarcomeres with a spatial periodicity, ie, sarcomere length $(l)$, ranging from 0.9 to $1.5 \mu \mathrm{m} .{ }^{27}$ Each sarcomere consists of actin-myosin structures bundled together in parallel such that there are 10-30 actin filaments across the diameter of the SF. ${ }^{8}$ Assuming the SF cross-section is circular, this corresponds to roughly 100-700 filaments per sarcomere.

A minimal model for an SF must describe both the passive stiffness and the active contractile properties of the SF. To account for these properties, individual actomyosin subunits are described mechanically as two actin filament springs connected by a myosin filament (Figure 1B). Russell et al ${ }^{28}$ recently provided experimental support for this mechanical description of SF sarcomeres by tracking the initial elastic recoil and subsequent contractile shortening of individual

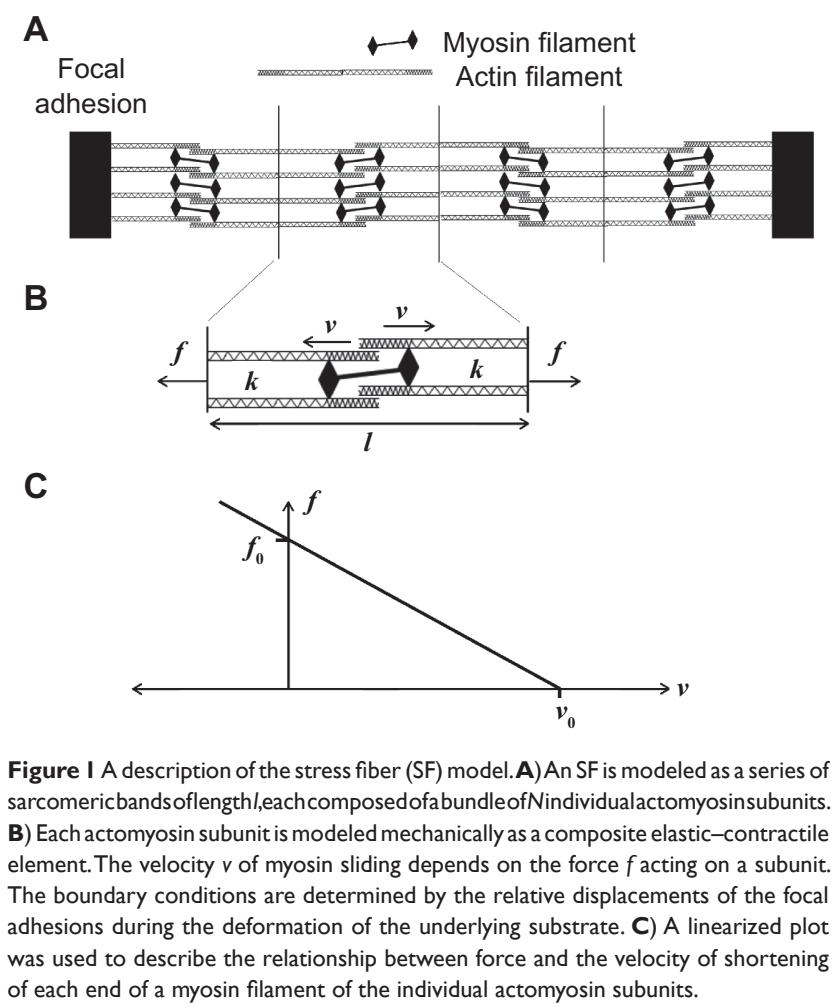


sarcomeres within severed SFs in living cells. We expand on their model to describe the time-dependent response of intact SFs to changes in length.

We have previously demonstrated that the force-strain relationship for isolated SFs under rigor conditions is approximately linear at physiological strains (ie, $F=K \varepsilon$ for $\varepsilon<0.3) .{ }^{29}$ As an initial approximation, the stiffness $K$ of a sarcomere is assumed to be proportional to the number of actomyosin subunits $N$ in the sarcomere, ie, $K=k N$, where $k$ represents the stiffness of each actomyosin subunit (Figure 1B). Note that the level of tension in the SF $(F)$ is equal to the force acting on (or, equivalently, generated by) the $N$ actomyosin subunits acting in parallel in an SF crosssection (ie, $f=F / N$ ). This stiffness is not attributed to any one component of the actomyosin subunit but represents the composite stiffness of an average actomyosin subunit. Further, the SF is assumed to be composed of a series of identical sarcomeres where the strain $\varepsilon$ in each sarcomere (and each actomyosin subunit) is equivalent to the strain in the entire SF. Noting that $\varepsilon=\left(l-l_{\text {ref }}\right) / l_{\text {ref }}$, the force on each actomyosin subunit $f$ is related to strain,

$$
f=k \frac{l-l_{\text {ref }}}{l_{\text {ref }}}
$$

where $l$ is the current length of the sarcomere. The reference length $l_{\text {ref }}$ is the length of the sarcomere if it were to suddenly elastically retract upon unloading (ie, when $f=0$ ). The regions of overhanging actin do not contribute to the reference length, as they are not under tension. Consequently, myosin movement along actin filaments at a shortening velocity $v$ results in a change in the reference length as the amount of overhanging actin changes,

$$
\frac{\mathrm{d} l_{\text {ref }}}{\mathrm{dt}}=-2 v
$$

where the factor of 2 comes from the additive shortening of each end of a myosin filament. Let us now consider the role of myosin sliding on the time-dependent behavior of the sarcomeres. Differentiation of Eq. 1 and substitution of Eq. 2 into the result gives

$$
\frac{\mathrm{d} f}{\mathrm{dt}}=\frac{k}{l_{\mathrm{ref}}} \frac{\mathrm{d} l}{\mathrm{dt}}+\frac{2 k l v}{l_{\mathrm{ref}}^{2}}
$$

The first term on the right side of the equation represents the contribution of the elasticity of the actomyosin subunit. The second term represents the rate that the sarcomere relaxes toward the stall force through myosin movement. The relative magnitude of these two terms determines the force generation in the SF. If the SF changes length much more quickly than myosin can respond, then the first term dominates and the SF behaves elastically with a force that increases in proportion to the length. If the SF changes length slowly, then the second term will be roughly equal and opposite in magnitude to the first term, and the force will remain nearly constant.

The velocity of myosin shortening depends on the force experienced by the myosin filament. Figure $1 \mathrm{C}$ illustrates schematically a linearized form of the force-velocity relationship first reported for skeletal muscle in the classic work of Hill. ${ }^{30}$ In this plot, $f_{0}$ is the stall force, and $v_{0}$ is the velocity of shortening when the actomyosin subunit is completely unloaded. This plot also illustrates eccentric contraction (ie, $v<0$ when $f>f_{0}$ ). Debold et $\mathrm{al}^{23}$ reported a similar force-velocity relationship for skeletal muscle myosin that interacted with actin-coated microbeads in an optical trap such that only eight myosin head groups could interact with actin at a time. These authors demonstrated that the microbeads moved with a negative velocity when the applied force exceeded the stall force through a process involving slipping and reattachment of myosin heads. ${ }^{23}$ Myosin filaments in SFs contain 8-10 myosin molecules on each end of the myosin filament, ${ }^{18,20}$ suggesting that SFs may also undergo lengthening via slipping and reattachment of myosin heads. Literature values for $v_{0}$ range from $300 \mathrm{~nm} / \mathrm{s}$ for nonmuscle myosin II in gliding assays ${ }^{31,32}$ to $\sim 10 \mathrm{~nm} / \mathrm{s}$ in intact SF sarcomeres. ${ }^{28}$ To our knowledge, the value for $f_{0}$ has not been reported for nonmuscle myosin II. Russell et $\mathrm{a}^{28}$ estimated values of 1.21 and $0.085 \mu \mathrm{m}$ for initial length and elastic retraction distance, respectively, for individual sarcomeres, which predicts an equilibrium prestrain of 0.076 . This is roughly consistent with the value of $\sim 0.10$ reported by $\mathrm{Lu}$ et al. ${ }^{15}$ The model parameters and relevant literature values are summarized in Table 1.

\section{Prestress and pre-extension of SFs in static cells}

Cells reportedly maintain an equilibrium cytoskeletal prestress $^{33}$ or pre-extension ${ }^{15}$ under static conditions. Under these conditions $(v=0)$, inspection of Eq. 3 reveals that the steady-state force on an actomyosin subunit is simply the stall force $f_{0}$, and the strain is $\varepsilon_{0}=f_{0} / k$. Assuming that the cross-sectional area of an actomyosin subunit $(A)$ remains unchanged under small strains, the model predicts that the SF maintains force per unit area, or stress, at a constant level equal to $f_{0} / A$. Given that the strain in an individual sarcomere is equal to that of the entire SF, the 
Table I Model parameters

\begin{tabular}{|c|c|c|c|c|}
\hline Parameters & Meaning & Values & Literature values & References \\
\hline$f_{0}$ & Myosin stall force & $17 \mathrm{pN}^{\mathrm{a}}$ & - & - \\
\hline$v_{0}$ & Maximum myosin velocity & $0.03 \mu \mathrm{m} / \mathrm{s}$ & $0.0 \mathrm{I}-0.3 \mu \mathrm{m} / \mathrm{s}$ & $28,31,32$ \\
\hline$L$ & Sarcomere length & $\mathrm{I} \mu \mathrm{m}$ & $0.9-1.5 \mu \mathrm{m}$ & 27 \\
\hline$K$ & SF spring constant & $49 \mathrm{nN}$ & $49 \mathrm{nN}^{\mathrm{b}}$ & 29 \\
\hline$N$ & Actomyosin subunits per sarcomere & - & $100-700^{c}$ & - \\
\hline$k$ & Actomyosin subunit spring constant & $170 \mathrm{pN}^{\mathrm{d}}$ & $70-490 \mathrm{pN}^{\mathrm{e}}$ & - \\
\hline$N_{\mathrm{SF}}$ & Number of SFs & 1000 & - & - \\
\hline$\Delta t$ & Time increment & $0.01 \mathrm{~s}$ & - & - \\
\hline$\alpha$ & SF disassembly parameter & $0.00015 \mathrm{~s}^{-1}$ & - & - \\
\hline$\beta$ & SF disassembly parameter & 0.06 & - & - \\
\hline$\gamma$ & SF disassembly parameter & $0.1 \mu \mathrm{m} / \mathrm{s}$ & - & - \\
\hline
\end{tabular}

Notes: abased on each myosin minifilament containing 10 myosin molecules per side and a stall force for muscle myosin of I.7 pN; ${ }^{35}$ b The force-strain curve is nearly linear for physiological strains $(\varepsilon<0.3)$; 'SFs contain 10-30 actin filaments across their diameter, ${ }^{8}$ suggesting that each SF consists of a bundle of I00-700 actin-myosin subunits assuming a circular cross-section; 'Based on equilibrium SF prestrain $\varepsilon_{0}=f_{0} / \mathrm{k}=0.10 ;{ }^{15} \mathrm{e}=K / N$.

Abbreviation: SF, stress fiber.

model predicts that SF strain is also maintained constant at equilibrium. Thus, the model indicates that prestress and pre-extension are mutually related and are maintained by myosin force balances at the sarcomere level.

\section{Cyclic stretching of an individual stress fiber}

Several cell types, particularly those residing in the heart, arteries, and lungs, are subject to cyclic stretch patterns. Cells and their SFs respond to cyclic stretch in a frequency-dependent manner, with different cells showing different sensitivities to frequency. ${ }^{26,34}$ Numerical solutions to Eqs. 2 and 3 were derived using the MATLAB (The MathWorks, Natick, MA, USA) algorithm ODE45, with the values for the model parameters inferred from the literature (Table 1). These results are illustrated by the harmonic response of the model as a function of frequency (Figure 2). The time axis is scaled by the period of oscillation to facilitate comparisons between responses at different stretch frequencies. Equation 3 provides insight into the mechanisms determining the sensitivity to the frequency of cyclic stretch. At high frequencies, the first term on the right side of the equation is much larger than the second term; hence, the amplitude in force is proportional to the amplitude in substrate strain. The average force, however, does gradually decrease to $f_{0}$. The force amplitude decreases as frequency decreases because the second term becomes increasingly important. At very low frequencies, almost no force is generated by substrate stretching. In addition, there is an obvious phase shift as the stretch frequency decreases, which is indicative of a viscous-like effect caused by myosin sliding. Note that the force in the SF is simply $F=f N$. Thus, the tension in an SF composed of $N$ actomyosin subunits in a cross-section follows the same pattern as that shown in Figure 2.
We next analyzed the sensitivity of the model response to the model parameters $f_{0}, k, v_{0}$, and $l$. A relative stiffness was calculated, which indicates the ratio of the force amplitude relative to that for a purely elastic model of stiffness $k$. In all cases, the curves showed a gradual increase in relative stiffness as frequency increased up to a saturation frequency above which the relative stiffness was unity. Increasing $f_{0}$ (Figure 3A) or decreasing $k$ (Figure 3B) caused a decrease in the saturation frequency. Decreasing $v_{0}$ from 300 to $10 \mathrm{~nm} / \mathrm{s}$ caused the greatest shift in saturation frequency (Figure 3C), with decreasing $v_{0}$ resulting in an increase in SF relative stiffness. The response was comparatively insensitive to changes in sarcomere length $l$ over the range of $0.9-1.5 \mu \mathrm{m}$ (Figure 3D), where $l$ represents the length of the sarcomere under the initial static conditions.

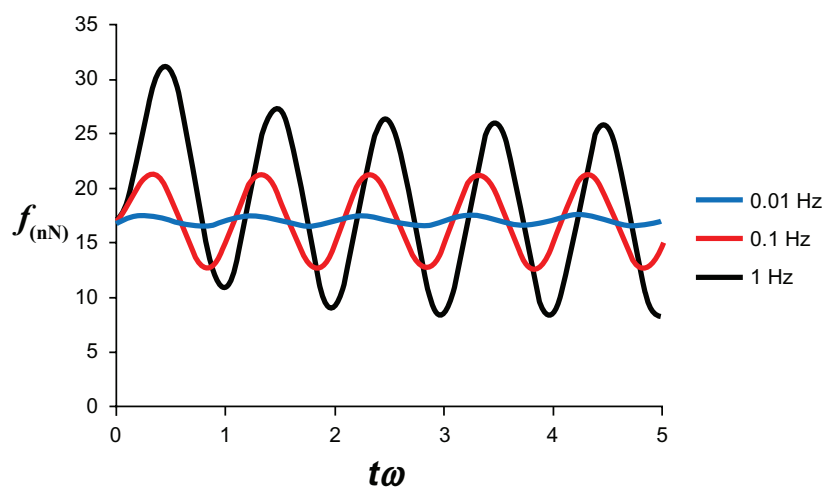

Figure 2 The response of an individual actomyosin subunit to cyclic stretch is frequency dependent. The predicted time course of force generation is shown for an actomyosin subunit in a stress fiber subject to sinusoidal strain of $10 \%$ amplitude and frequencies of I (black curve), 0.1 (red curve), and $0.01 \mathrm{~Hz}$ (blue curve). The time axis is normalized by frequency $\omega$ to facilitate comparison between results at different frequencies. 

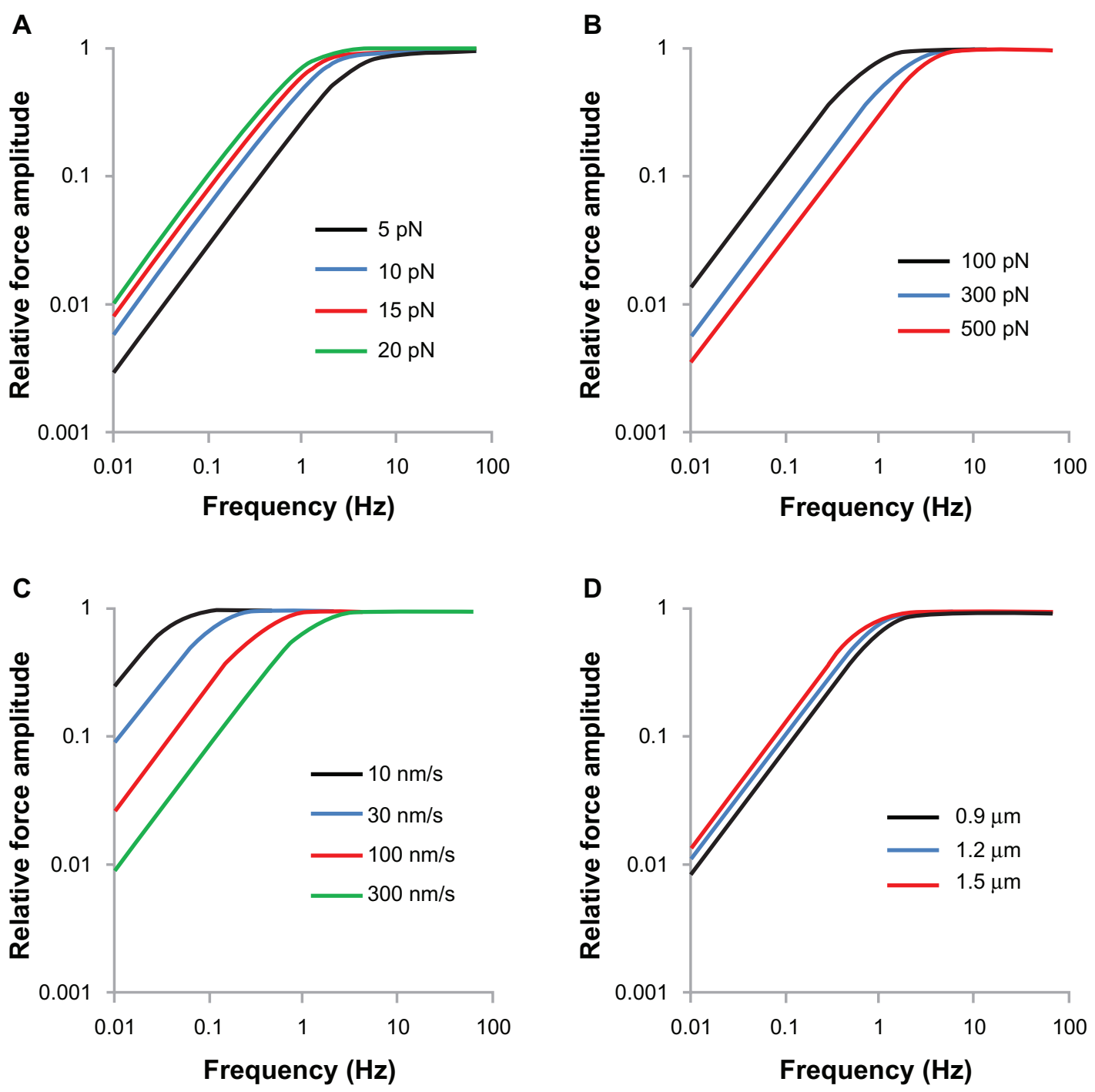

Figure 3 The response of the stress fiber (SF) model depends on the model parameter values. The plots illustrate the effects of $\mathbf{A}) f_{0}$, B) $k$, C) $v_{0}$, and $\left.\mathbf{D}\right) /$ on the relationship between relative force amplitude and stretch frequency. The relative force amplitude is the ratio of the amplitude of force divided by the force that would be generated for a purely elastic SF. As a basis, the parameter values used were $f_{0}=17 \mathrm{pN}, v_{0}=300 \mathrm{~nm} / \mathrm{s}, I=I .0 \mu \mathrm{m}$, and $k=I 70 \mathrm{pN}$. The model was solved by varying each parameter individually to determine the sensitivity of the response to changes in the parameter.

\section{Frequency-dependent SF reorganization}

We next considered the effects of myosin sliding on SF stability and the resulting reorganization of an SF network into a new steady-state organization upon application of cyclic stretch at different frequencies and patterns. Rapid crossbridge cycling, such as occurs during concentric loading, is predicted to lower the fraction of bound myosin heads, ie, the duty ratio. ${ }^{19}$ As described by Howard, ${ }^{35}$ the duty ratio is also equal to the ratio of the length of a power stroke and the distance between binding sites along the actin filament. Myosin binds to actin at sites $\sim 36 \mathrm{~nm}$ apart, ${ }^{36}$ which is much larger than the myosin head working distance. Thus, each myosin head must wait until the actin filament has traveled some integer multiple of $36 \mathrm{~nm}$ before another binding event can occur. Further, myosin may miss some potential binding sites, depending on how quickly these sites pass by. Howard ${ }^{35}$ expressed the probability of myosin missing any particular binding site increases as the velocity of actin passing the myosin head increases as $\exp \left(-k_{0} x_{0} / v\right)$, where $k_{0}$ is the binding rate, and $x_{0}$ represents the width of the binding site "window". Therefore, increasing myosin velocity decreases the fraction of bound myosin heads. We propose that the probability of SF disassembly over a time interval $\Delta t$ is also related to myosin velocity through a similar relationship:

$P($ disassembly of the $i$ th SF $)=\left[\alpha+\beta \exp \left(-\frac{\gamma}{|v|}\right)\right] \Delta t$ 
where $\alpha, \beta$, and $\gamma$ are model parameters. Myosin slipping caused by applying forces greater than the stall force is reported to involve transient detachment of actomyosin crossbridges with more detachments occurring as force increases. ${ }^{23}$ Therefore, myosin movement caused by either concentric or eccentric contraction (positive or negative velocity) is expected to reduce the duty ratio and hence decrease the stability of an SF due to fewer actomyosin cross-links. Disassembled SFs are assumed to provide the building material for the assembly of new SFs. Although SF assembly is expected to take seconds to minutes, in the present analysis, each disassembled SF is assumed to reassemble immediately. It is not clear how cyclic stretch determines the orientation of newly assembling fibers; hence, the newly assembled SF is oriented in a randomly chosen direction at an initial strain $\varepsilon_{0}$.

The model was solved using $\mathrm{C}++$ code as previously described ${ }^{37}$ with modifications to incorporate Eqs. 2-4. The initial conditions were that the orientations of the population of the $N_{\mathrm{SF}}$ SFs were taken to be randomly distributed with each SF having an initial strain $\varepsilon_{0}$. This initial strain represents the pre-elongation caused by cell contractility. Further changes in SF strain occur through deformation of the underlying matrix. The temporal pattern of matrix deformation was described as a series of incremental stretches taken over time increments $\Delta t$. Sinusoidal changes in substrate strain were applied in the $x$-direction with the strain in the $y$-direction dependent on the Poisson ratio of the substrate $\left(-\varepsilon_{y} / \varepsilon_{x}\right)$. Figure 4 illustrates the calculated temporal evolution of SF orientations following $10 \%$ cyclic pure uniaxial stretch $\left(\varepsilon_{x}=0.10, \varepsilon_{y}=0\right)$ at frequencies ranging from 0.01 to $1 \mathrm{~Hz}$. The model was solved using the parameter values listed in Table 1. To characterize the dispersion in SF orientations, we computed the circular variance in the distribution,

Circular variance $=1-\frac{1}{N_{\mathrm{SF}}} \sqrt{\left(\sum_{i=1}^{N_{\mathrm{SF}}} \sin 2 \theta^{j}\right)^{2}+\left(\sum_{i=1}^{N_{\mathrm{SF}}} \cos 2 \theta^{j}\right)^{2}}$

where $\theta^{i}$ is the orientation of the $i$ th SF. As these are axial vectors, the angles must be multiplied by a factor of two to transform the distribution into a range of $0^{\circ}-360^{\circ} .{ }^{38}$ The values range from zero to unity, corresponding to perfect alignment and a uniform distribution, respectively. At $0.01 \mathrm{~Hz}$, the SF distribution remains random at all time points, as tension on myosin filaments is maintained at $f_{0}$ due to myosin sliding. As the frequency increases, the extent of SF alignment perpendicular to the stretch direction increases. There is no additional SF alignment when the stretch frequency is raised above a saturation frequency of $\sim 1 \mathrm{~Hz}$. At and above this saturation frequency, the rate of change in sarcomere length is much faster than the velocity of myosin sliding; hence, the SF behaves nearly elastically. These results compare well with experimentally measured values of circular variance of SF orientation distributions measured in endothelial cells subject to these same stretch conditions. ${ }^{26}$

SFs in cells subjected to stretch with lateral thinning (ie, the Poisson effect) align obliquely to the principal direction of

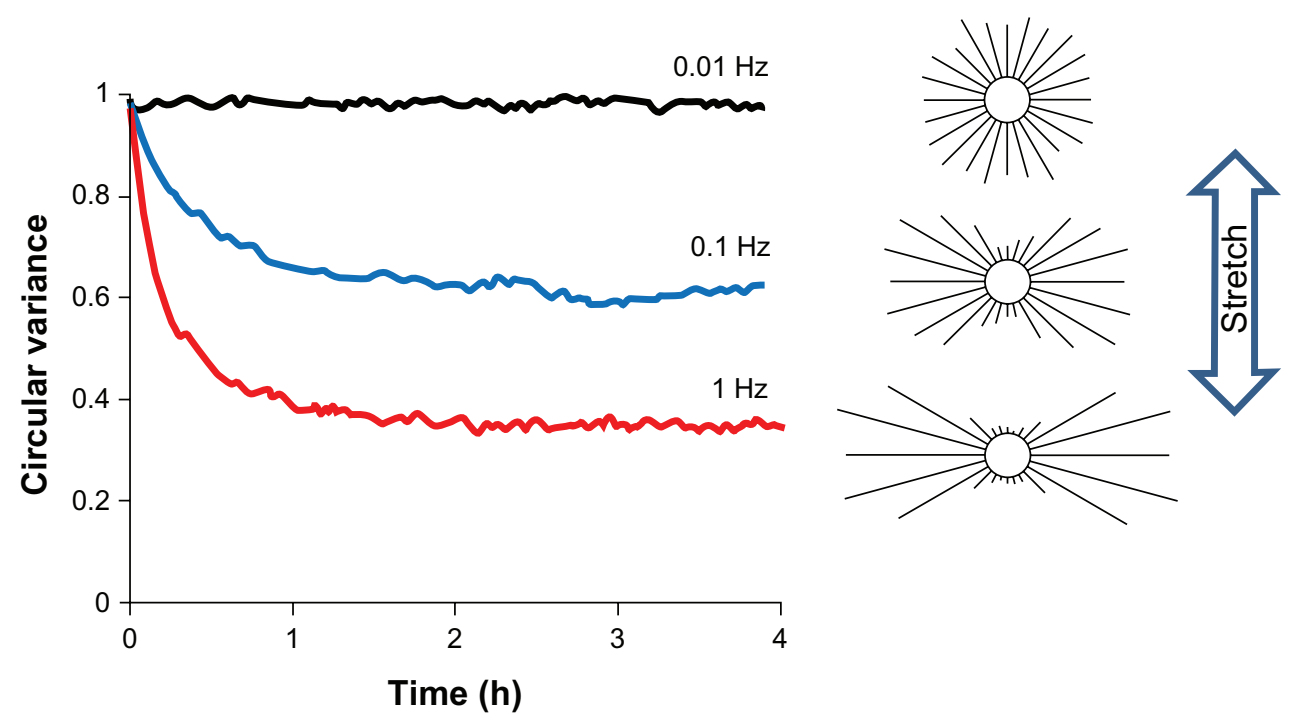

Figure 4 Predicted time course of stress fiber (SF) reorientation in response to pure uniaxial cyclic stretch. The circular variances of the distribution in SF orientations were calculated for 10\% pure uniaxial stretch at $0.0 \mathrm{I}-\mathrm{I} \mathrm{Hz}$. The distributions of SF orientations are shown after $4 \mathrm{~h}$ of stretching. The values for model parameters used in this solution are listed in Table I. 
stretch. ${ }^{24,25}$ Specifically, for $10 \%$ uniaxial stretch at $1 \mathrm{~Hz}$ in the $x$-direction with lateral shortening in the $y$-direction defined by the Poisson ratio of the substrate $\left(-\varepsilon_{y} / \varepsilon_{x}=0.35\right)$, the SFs aligned at an angle of $\pm 60^{\circ}$ relative to the $x$-direction (Figure 5). This prediction corresponds well with the measured angle of $\pm 60^{\circ}$ reported for endothelial cells subject to $10 \%$ cyclic stretch on silicone rubber membranes where $v=0.35 .{ }^{39}$ The SFs within individual cells tend to be roughly coaligned in our experience; thus, the bimodal distribution should be interpreted as approximately half of the cells in a population predicted to have SFs aligned $60^{\circ}$ from the direction of stretch, while the other half contain SFs aligned $-60^{\circ}$ from the direction of stretch. As the SFs aligned in two different axial directions, circular variance is a poor metric for alignment and was not calculated.

\section{Discussion}

Individual cells tend to maintain intracellular strain ${ }^{15}$ and stiffness $^{33}$ at a particular level. Mizutani et $\mathrm{al}^{40}$ demonstrated that

$0.01 \mathrm{~Hz}$

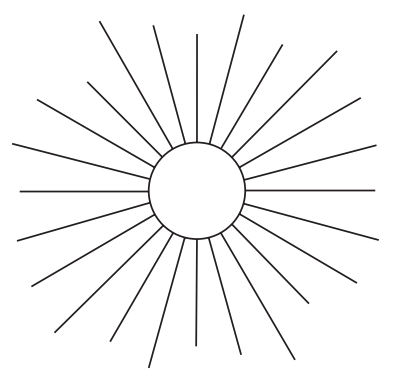

$0.1 \mathrm{~Hz}$
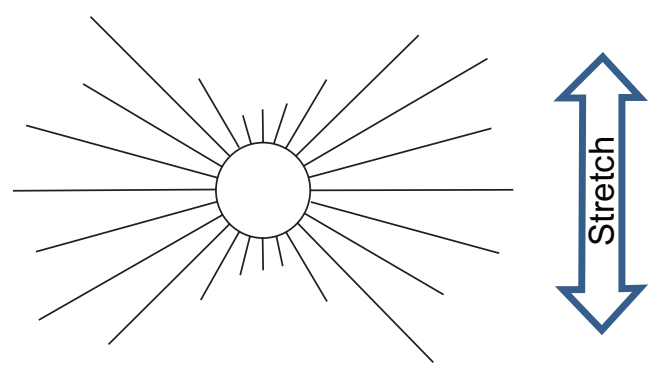

$1 \mathrm{~Hz}$

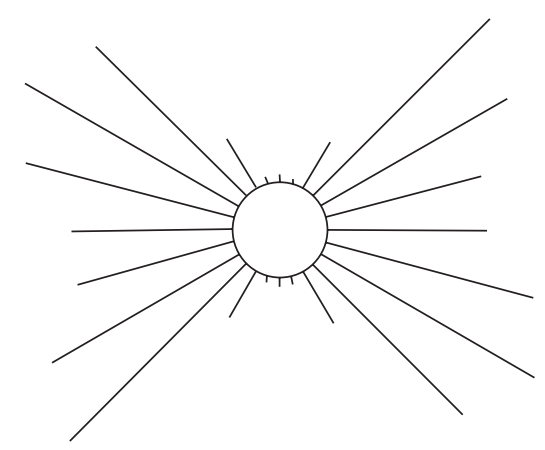

Figure 5 Predicted frequency dependence of stress fiber (SF) reorientation in response to simple uniaxial cyclic stretch. The distributions in SF orientations were calculated for $4 \mathrm{~h}$ of $10 \%$ uniaxial stretch at $0.0 \mathrm{I}-\mathrm{I} \mathrm{Hz}$ of an elastic substrate with a Poisson ratio of 0.35. The values for model parameters used in this solution are listed in Table I. cell stiffness relaxes following an initial increase in stiffness caused by cell stretching. They attributed this tensional homeostasis to SFs but did not propose a specific mechanism by which tensional regulation occurs. In the current study, we analyzed tension regulation in individual SFs using a model based on the Hill-type force-velocity relationship for myosin sliding. This model predicts that prestrain and prestress are generated by the steady-state balance between myosin filaments and elastic resistance to stretching of the actomyosin subunits. Further, the model explains how SFs can regulate tension through two different mechanisms that become important under different situations. Under conditions when the rate of strain is low, such as during cyclic stretching at low stretch frequencies, myosin sliding quickly readjusts the reference length of the SF so as to quickly re-establish tensional homeostasis. Under conditions when the SF is subject to high rates of strain, such as during cyclic stretching at high stretch frequencies, myosin sliding is much slower than the rate of change in sarcomere length, and the SF behaves elastically. Here, the SFs must orient away from the direction of stretch in order to minimize perturbations in tension. This realignment process occurs as a consequence of the asymmetric increase in the rate of disassembly of SFs undergoing high rates of change in length, resulting in the accumulation of SFs in orientations that avoid changes in length. Thus, both relaxation and reorientation are important mechanisms that cells use to maintain SF tension at a preferred level, and myosin II plays an important role in each of these activities.

There is accumulating evidence that SF tension has a large impact on cell function. Increased myosin activity via Rho GTPase activation has been linked to transformation of cells into a malignant phenotype ${ }^{7}$ and directs the differentiation of mesenchymal stem cells into an osteoblast fate. ${ }^{2}$ Our model predicts that the level of SF tension is dependent on myosin stall force $f_{0}$ and the number of actomyosin subunits $N$ in the cross-section of the SF. The mechanisms that regulate the value of $f_{0}$ for a given cell are not clear but are likely dependent on myosin ATPase activity. Jungbauer et $\mathrm{al}^{34}$ reported that rat embryonic fibroblasts and human fibroblasts exhibit threshold frequencies for stretch-induced alignment of 0.01 and $0.1 \mathrm{~Hz}$, respectively. It remains to be determined whether these differences can be attributed to differences in the properties of SFs in these two cell lines. Our analysis indicates that the frequency dependence of cyclic stretch-induced SF alignment depends on factors that regulate the myosin force-velocity relationship, sarcomere dimensions, and actomyosin subunit stiffness (Figure 3). 
Brown et al ${ }^{16}$ first used the term "tensional homeostasis" to describe the behavior of fibroblasts residing within cyclically loaded collagen gels, noting that increased external loading was followed immediately by a reduction in cell-mediated contraction, whereas decreased external loading elicited increased contraction. Wakatsuki et $\mathrm{al}^{41}$ and Zahalak et $\mathrm{al}^{42}$ applied harmonic testing of fibroblast-seeded collagen gels and reported that the elastic modulus increases with $\log (\omega)$ for frequencies ranging from 0.008 to $0.4 \mathrm{~Hz}$. This is consistent with the current results (Figure 3). Importantly, activating the cells with fetal calf serum caused the elastic modulus and phase angle to increase at all frequencies, which the authors attributed to increased myosin activity. Thus, myosin activity contributes to the overall stiffness and relaxation response of reconstituted tissue equivalents.

The time-dependent mechanical properties of functional SFs have been estimated by severing SFs within intact cells and tracking their rate of recoil. ${ }^{28,43,44}$ Mechanical models were proposed to interpret the measurements from these experiments where individual sarcomeres were modeled as either springs in parallel with a contractile element and a dashpot ${ }^{43,45}$ or a spring in series with a contractile element. ${ }^{28} \mathrm{~A}$ spring in parallel with a contractile element would not be fully unloaded at low strain rates, leading to asymmetric increases in tension of SFs in the direction of stretch even at very low stretch frequencies. This is inconsistent with our experimental results that SFs do not respond to stretching at $0.01 \mathrm{~Hz} \cdot{ }^{26}$ However, a spring in series with a contractile element would remain at equilibrium at low strain rates. This is very similar to our model of an actomyosin subunit; however, in our model, the elastic and contractile elements are not treated as separate elements of an actomyosin subunit. Instead, myosin sliding acts to change the reference length of the elastic element so that strain changes without a change in the current length of the element (ie, the actomyosin subunit). Na et $\mathrm{al}^{46}$ modeled actin cytoskeletal remodeling in response to cyclic equibiaxial strain based solely on SF turnover, but their model cannot account for $\mathrm{SF}$ realignment or strain-rate dependence. De et a ${ }^{47}$ modeled stretch-induced cell alignment by treating whole cells as force dipoles. Similar to the current model, they proposed that cells try to maintain a mechanical variable at a set point level, but in their model the variable was the level of stress or strain in the adjacent elastic matrix. The present model seeks to provide insight into the role of myosin II, which we predict mediates both SF relaxation and turnover. Each of these phenomena contributes to SF adaptation to cyclic stretch, with SF relaxation being important at low strain rates and SF turnover becoming important as strain rate increases.
Our model implicates actomyosin crossbridge cycling as the source for the apparent viscoelastic behavior of SFs. This mechanism is supported by direct measurements of SF sarcomere shortening after SF severing. ${ }^{28}$ However, we cannot rule out other potential mechanisms that may also contribute to SF relaxation. Cytoskeletal fluidization has been proposed as a mechanism for stress dissipation in stretched cells. ${ }^{48}$ However, we have not observed the fluidization of SFs in cells expressing GFP-actin subject to $0.01 \mathrm{~Hz}$ cyclic stretch. ${ }^{37,49}$ Myosin light chain and $\alpha$-actinin turnover rates are reported to be in the range of $1-3 / \mathrm{min}$ in U2OS cells. Thus, the unbinding of stressed SF proteins followed by their rebinding in an unstressed configuration may relieve the overall stress in the SF. It is likely that sarcomere mechanics and SF protein turnover each play a role, and future studies using techniques such as fluorescence recovery after photobleaching of fluorescently labeled SFs subject to cyclic stretch will need to be performed to assess the role of SF protein turnover in SF relaxation.

Several simplifying assumptions were used to maintain the mathematical transparency of the current model without sacrificing physiological relevance. Sarcomere dimensions and actomyosin subunit force-velocity relationships are assumed to be uniform throughout an SF. There is evidence, however, that the contractile response of sarcomeres can vary along the length of an $\mathrm{SF}^{50}$ There is also some variance in sarcomere contraction distance in freely shortening SFs. ${ }^{28}$ Another limitation to our model is that it lacks sensitivity to biochemical inputs. In particular, it does not directly address regulation of myosin activity by signaling via myosin light chain kinases. The increase in elastic modulus and phase angle reported by Wakatsuki et $\mathrm{al}^{41}$ in response to stimulation with fetal calf serum suggests that increased myosin activation will increase both stall force and myosin shortening velocity. There is a need for more direct measurement of the effect of myosin activation on the force-velocity relationship of SFs or sarcomeres. The turnover of SFs does not include a description of SF assembly kinetics. Instead, SFs simply reassemble immediately after disassembling. The rebuilding of focal adhesions is not considered in the present study. Recently, we demonstrated that cyclic stretch-induced SF reorientation did not require the turnover or sliding of associated focal adhesions. ${ }^{49}$ Finally, our model does not capture the details of the complex process by which SFs reassemble during reorientation. Nonetheless, the present model captures many of the salient features of stretch-induced SF reorganization using rules motivated by the effects of actomyosin interactions. 


\section{Conclusion}

Although quantitative modeling does not provide direct evidence for a hypothetical mechanism, it is a powerful tool for testing whether the hypothesis is sufficient to explain experimental observations and to generate new hypotheses. This approach is particularly useful for elucidating the complex interactions in a dynamic and complex system, which can be difficult or misleading to predict based on intuition. The model presented here provides a framework for incorporating molecular details of myosin II behavior into mechanical models of SFs and SF networks. There is accumulating evidence that cellular regulation of intracellular tension through SF relaxation and reorientation is important in maintaining not only mechanical homeostasis of the cytoskeleton but also downstream mechanotransduction. ${ }^{37}$ Experiments guided by and interpreted using such models promise to shed light on the complex interplay of intra- and extracellular forces in regulating cell shape and function.

\section{Acknowledgment}

The author was supported by grants from the American Heart Association (0730238N) and National Science Foundation (CBET-0854129) to RK.

\section{Disclosure}

The authors report no conflicts of interest in this work.

\section{References}

1. Kaunas R, Nguyen P, Usami S, Chien S. Cooperative effects of Rho and mechanical stretch on stress fiber organization. Proc Natl Acad Sci US A. 2005;102(44):15895-15900.

2. McBeath R, Pirone DM, Nelson CM, Bhadriraju K, Chen CS. Cell shape, cytoskeletal tension, and RhoA regulate stem cell lineage commitment. Dev Cell. 2004;6(4):483-495.

3. Hinz B, Mastrangelo D, Iselin CE, Chaponnier C, Gabbiani G Mechanical tension controls granulation tissue contractile activity and myofibroblast differentiation. Am J Pathol. 2001;159(3): 1009-1020.

4. Park JS, Chu JS, Cheng C, Chen F, Chen D, Li S. Differential effects of equiaxial and uniaxial strain on mesenchymal stem cells. Biotechnol Bioeng. 2004;88(3):359-368.

5. Assoian RK, Klein EA. Growth control by intracellular tension and extracellular stiffness. Trends Cell Biol. 2008;18(7):347-352.

6. Nelson CM, Jean RP, Tan JL, et al. Emergent patterns of growth controlled by multicellular form and mechanics. Proc Natl Acad Sci US A. 2005;102(33):11594-11599.

7. Paszek MJ, Zahir N, Johnson KR, et al. Tensional homeostasis and the malignant phenotype. Cancer Cell. 2005;8(3):241-254.

8. Cramer LP, Siebert M, Mitchison TJ. Identification of novel graded polarity actin filament bundles in locomoting heart fibroblasts: implications for the generation of motile force. J Cell Biol. 1997;136(6): 1287-1305.

9. Lazarides E, Burridge K. Alpha-actinin: immunofluorescent localization of a muscle structural protein in nonmuscle cells. Cell. 1975;6(3): 289-298.
10. Langanger G, Moeremans M, Daneels G, Sobieszek A, De Brabander M, De Mey J. The molecular organization of myosin in stress fibers of cultured cells. J Cell Biol. 1986;102(1):200-209.

11. Burridge K, Fath K, Kelly T, Nuckolls G, Turner C. Focal adhesions: transmembrane junctions between the extracellular matrix and the cytoskeleton. Annu Rev Cell Biol. 1988;4:487-525.

12. Hotulainen P, Lappalainen P. Stress fibers are generated by two distinct actin assembly mechanisms in motile cells. J Cell Biol. 2006; 173(3):383-394.

13. Polte TR, Eichler GS, Wang N, Ingber DE. Extracellular matrix controls myosin light chain phosphorylation and cell contractility through modulation of cell shape and cytoskeletal prestress. Am J Physiol Cell Physiol. 2004;286(3):C518-C528.

14. Deguchi S, Ohashi T, Sato M. Evaluation of tension in actin bundle of endothelial cells based on preexisting strain and tensile properties measurements. Mol Cell Biomech. 2005;2(3):125-133.

15. Lu L, Feng Y, Hucker WJ, Oswald SJ, Longmore GD, Yin FC. Actin stress fiber pre-extension in human aortic endothelial cells. Cell Motil Cytoskeleton. 2008;65(4):281-294.

16. Brown RA, Prajapati R, McGrouther DA, Yannas IV, Eastwood M. Tensional homeostasis in dermal fibroblasts: mechanical responses to mechanical loading in three-dimensional substrates. J Cell Physiol. 1998;175(3):323-332.

17. Wilson CA, Tsuchida MA, Allen GM, et al. Myosin II contributes to cell-scale actin network treadmilling through network disassembly. Nature. 2010;465(7296):373-377.

18. Kovács M, Thirumurugan K, Knight PJ, Sellers JR. Load-dependent mechanism of nonmuscle myosin 2. Proc Natl Acad Sci U S A. 2007 104(24):9994-9999.

19. Howard J. Molecular motors: structural adaptations to cellular functions. Nature. 1997;389(6651):561-567.

20. Vicente-Manzanares M, Ma X, Adelstein RS, Horwitz AR. Non-muscle myosin II takes centre stage in cell adhesion and migration. Nat Rev Mol Cell Biol. 2009;10(11):778-790.

21. Uyeda TQ, Kron SJ, Spudich JA. Myosin step size. Estimation from slow sliding movement of actin over low densities of heavy meromyosin. J Mol Biol. 1990;214(3):699-710.

22. Matsui TS, Ito K, Kaunas R, Sato M, Deguchi S. Actin stress fibers are at a tipping point between conventional shortening and rapid disassembly at physiological levels of MgATP. Biochem Biophys Res Commun. 2010;395(3):301-306

23. Debold EP, Patlak JB, Warshaw DM. Slip sliding away: loaddependence of velocity generated by skeletal muscle myosin molecules in the laser trap. Biophys J. 2005;89(5):L34-L36.

24. Wang JH. Substrate deformation determines actin cytoskeleton reorganization: a mathematical modeling and experimental study. J Theor Biol. 2000;202(1):33-41.

25. Yamada H, Takemasa T, Yamaguchi T. Theoretical study of intracellular stress fiber orientation under cyclic deformation. J Biomech. 2000; 33(11):1501-1505.

26. Hsu HJ, Lee CF, Kaunas R. A dynamic stochastic model of frequencydependent stress fiber alignment induced by cyclic stretch. PLoS One 2009;4(3):e4853.

27. Sanger JW, Sanger JM, Jockusch BM. Differences in the stress fibers between fibroblasts and epithelial cells. J Cell Biol. 1983;96(4): 961-969.

28. Russell RJ, Xia SL, Dickinson RB, Lele TP. Sarcomere mechanics in capillary endothelial cells. Biophys J. 2009;97(6):1578-1585.

29. Deguchi S, Ohashi T, Sato M. Tensile properties of single stress fibers isolated from cultured vascular smooth muscle cells. J Biomech. 2006; 39(14):2603-2610.

30. Hill AV. The heat of shortening and the dynamic constants of muscle Proc R Soc Lond B Biol Sci. 1938;126:136-195.

31. Hu A, Wang F, Sellers JR. Mutations in human nonmuscle myosin IIA found in patients with May-Hegglin anomaly and Fechtner syndrome result in impaired enzymatic function. J Biol Chem. 2002; 277(48):46512-46517. 
32. Wang F, Harvey EV, Conti MA, Wei D, Sellers JR. A conserved negatively charged amino acid modulates function in human nonmuscle myosin IIA. Biochemistry. 2000;39(18):5555-5560.

33. Wang N, Tolić-Nørrelykke IM, Chen J, et al. Cell prestress. I. Stiffness and prestress are closely associated in adherent contractile cells. $\mathrm{Am} \mathrm{J}$ Physiol Cell Physiol. 2002;282(3):C606-C616.

34. Jungbauer S, Gao H, Spatz JP, Kemkemer R. Two characteristic regimes in frequency-dependent dynamic reorientation of fibroblasts on cyclically stretched substrates. Biophys J. 2008;95(7):3470-3478.

35. Howard J. Mechanisms of Motor Proteins and the Cytoskeleton. Sunderland (MA): Sinauer Associates; 2001.

36. Molloy JE, Burns JE, Sparrow JC, Tregear RT, Kendrick-Jones J, White DC. Single-molecule mechanics of heavy meromyosin and S1 interacting with rabbit or Drosophila actins using optical tweezers. Biophys J. 1995;68(Suppl 4):298S-303S; 303S-305S.

37. Hsu HJ, Lee CF, Locke A, Vanderzyl SQ, Kaunas R. Stretch-induced stress fiber remodeling and the activations of JNK and ERK depend on mechanical strain rate, but not FAK. PLoS One. 2010;5(8):e12470.

38. Mardia KV, Jupp PE. Directional Statistics. 2nd ed. Chichester (UK): John Wiley and Sons Ltd; 2000.

39. Wang JH, Goldschmidt-Clermont P, Wille J, Yin FC. Specificity of endothelial cell reorientation in response to cyclic mechanical stretching. J Biomech. 2001;34(12):1563-1572.

40. Mizutani T, Haga H, Kawabata K. Cellular stiffness response to external deformation: tensional homeostasis in a single fibroblast. Cell Motil Cytoskeleton. 2004;59(4):242-248.

41. Wakatsuki T, Kolodney MS, Zahalak GI, Elson EL. Cell mechanics studied by a reconstituted model tissue. Biophys J. 2000;79(5): 2353-2368.
42. Zahalak GI, Wagenseil JE, Wakatsuki T, Elson EL. A cell-based constitutive relation for bio-artificial tissues. Biophys J. 2000;79(5): 2369-2381.

43. Colombelli J, Besser A, Kress H, et al. Mechanosensing in actin stress fibers revealed by a close correlation between force and protein localization. J Cell Sci. 2009;122(Pt 10):1665-1679.

44. Kumar S, Maxwell IZ, Heisterkamp A, et al. Viscoelastic retraction of single living stress fibers and its impact on cell shape, cytoskeletal organization, and extracellular matrix mechanics. Biophys J. 2006; 90(10):3762-3773.

45. Stachowiak MR, O’Shaughnessy B. Recoil after severing reveals stress fiber contraction mechanisms. Biophys J. 2009;97(2):462-471.

46. Na S, Meininger GA, Humphrey JD. A theoretical model for F-actin remodeling in vascular smooth muscle cells subject to cyclic stretch. J Theor Biol. 2007;246(1):87-99.

47. De R, Zemel A, Safran SA. Dynamics of cell orientation. Nat Phys. 2007;3:655-659.

48. Trepat X, Deng L, An SS, et al. Universal physical responses to stretch in the living cell. Nature. 2007;447(7144):592-595.

49. Lee CF, Haase C, Deguchi S, Kaunas R. Cyclic stretch-induced stress fiber dynamics - dependence on strain rate, Rho-kinase and MLCK. Biochem Biophys Res Commun. 2010;401(3):344-349.

50. Peterson LJ, Rajfur Z, Maddox AS, et al. Simultaneous stretching and contraction of stress fibers in vivo. Mol Biol Cell. 2004;15(7): 3497-3508.
Cell Health and Cytoskeleton

\section{Publish your work in this journal}

Cell Health and Cytoskeleton is an international, peer-reviewed open access journal focusing on all aspects of cell structure and function contributing to normal physiology and cell health and exploring the pathogenesis of cell dysfunction leading to adverse conditions and disease in the organism. The journal welcomes papers covering original research,

\section{Dovepress}

basic science, reviews and evaluations, guidelines, expert opinion and commentary, case reports and extended reports. The manuscript management system is completely online and includes a very quick and fair peerreview system, which is all easy to use. Visit http://www.dovepress.com/ testimonials.php to read real quotes from published authors. 